\title{
Neisseria meningitidis Induced Fatal Waterhouse-Friderichsen Syndrome in a Patient Presenting With Disseminated Intravascular Coagulation and Multiple Organ Failure
}

\author{
Meng-Yu Wu ${ }^{1,2} \mathbb{D}$, Chien-Sheng Chen ${ }^{1,2}$, Chih-Yi Tsay ${ }^{1,2}$, Giou-Teng Yiang ${ }^{1,2}$, Jian-Yu Ke ${ }^{1,2, *}$ \\ and Po-Chen Lin 1,2,* \\ 1 Department of Emergency Medicine, Taipei Tzu Chi Hospital, Buddhist Tzu Chi Medical Foundation, \\ New Taipei City 231, Taiwan; skyshangrila@gmail.com (M.-Y.W.); holeyeye@yahoo.com.tw (C.-S.C.); \\ belle99311101@gmail.com (C.-Y.T.); gtyiang@gmail.com (G.-T.Y.) \\ 2 Department of Emergency Medicine, School of Medicine, Tzu Chi University, Hualien 970, Taiwan \\ * Correspondence: ck4301018@gmail.com (J.-Y.K.); taipeitzuchier@gmail.com (P.-C.L.); \\ Tel.: +886-2-6628-9779 (J.-Y.K. \& P.-C.L.); Fax: +886-2-6628-9009 (J.-Y.K. \& P.-C.L.)
}

Received: 14 February 2020; Accepted: 15 March 2020; Published: 17 March 2020

\begin{abstract}
Neisseria meningitidis-induced acute systemic meningococcal disease is an emergency and a fatal condition that has a high mortality rate. In patients with a fulminant infection, a maculopapular petechial eruption, purpura fulminans, or an ecchymotic lesion are worrisome signs reflecting disseminated intravascular coagulation (DIC) and hint at Waterhouse-Friderichsen syndrome (WFS). Here, we describe a rare case of a patient with a fulminant Neisseria meningitidis-induced acute systemic meningococcal disease presenting with high-grade fever without meningitis symptoms. Fatal septicemia with DIC and multiple organ failure was noted. WFS was chiefly suspected. We highlight the clinical features and pathogenesis of Neisseria meningitidis-induced meningococcemia and WFS. We propose that they should be kept in mind, especially in patients presenting with a petechial eruption and purpura fulminans.
\end{abstract}

Keywords: Neisseria meningitidis; meningococcemia; Waterhouse-Friderichsen syndrome; disseminated intravascular coagulation; multiple organ failure

\section{Introduction}

Neisseria meningitidis-induced acute systemic meningococcal disease is an emergency and a fatal condition that has a high mortality rate [1]. Clinical manifestations of meningococcal meningitis and/or accompanying meningococcemia are commonly seen in patients with Neisseria meningitidis infection. However, absence of clinical evidence of meningitis in patients presenting with meningococcemia may lead to missed diagnosis and lack of timely treatment. In patients with a fulminant infection, a maculopapular petechial eruption, purpura fulminans, or an ecchymotic lesion may be seen. Severe sepsis may also induce disseminated intravascular coagulation (DIC) and Waterhouse-Friderichsen syndrome (WFS) described as acute hemorrhagic necrosis of the adrenal glands. These rare clinical features predict high morbidity and mortality [2]. Even with timely intervention, patients with Neisseria meningitidis-induced WFS usually die within $24 \mathrm{~h}$. Therefore, early diagnosis is very important for physicians for timely intervention in Neisseria meningitidis-induced acute systemic meningococcal disease and administration of antibiotics. Here, we describe a rare case of a fulminant Neisseria meningitidis-induced acute systemic meningococcal disease presenting with meningococcemia without meningitis. The patient had severe sepsis-induced DIC and multiple organ failure. WFS was chiefly 
suspected. The clinical features and pathogenesis of Neisseria meningitidis meningococcemia and WFS are discussed.

\section{Case Presentation}

A 67-year-old man presented with high-grade fever $\left(39.0^{\circ} \mathrm{C}\right)$ for a day. He had a past medical history of hypertension and cardiac arrhythmia, but denied having a history of diabetes mellitus, immunosuppression, or malignancy. Initial symptoms included high-grade fever and general muscle soreness. The patient did not have a headache, dyspnea, nausea, vomiting, abdominal pain, diarrhea, dysuria, chest pain, or a skin rash. Additional symptoms, such as hemorrhagic tendency/abnormality or arthritis, were not found. He had a history of traveling to Kinmen, Taiwan. However, he did not come in contact with other people with similar symptoms. On physical examination, his temperature was $39^{\circ} \mathrm{C}$, blood pressure was $142 / 85 \mathrm{~mm} \mathrm{Hg}$, heart rate was 101 beats/min, and the Glasgow Coma Score (GCS) was $\mathrm{E}_{4} \mathrm{~V}_{5} \mathrm{M}_{6}$. The breath sound was clear, and abdomen was soft without local tenderness. There were no abnormal skin lesions or neurological signs. The initial laboratory evaluation revealed no significant infection pattern, and the influenza test was negative (Table 1). The chest X-ray showed no significant pneumonia patch (Figure 1A). During observation at the ER, there were no progressive dyspnea or red flag signs. He received supportive care and was discharged. However, one day later, he revisited our hospital due to persistent high-grade fever and new onset of subconjunctival hemorrhage with petechial skin rash over the trunk and extremities. Other symptoms included epigastric pain and vomiting. Shock was suspected on the vital signs assessment: temperature was $38.8^{\circ} \mathrm{C}$, blood pressure was 107/66 mm Hg, and heart rate was 98 beats/min. The secondary laboratory evaluation revealed severe sepsis, acute kidney injury, and metabolic acidosis. Low platelet count, prolonged prothrombin time (PT), and activated partial thromboplastin time (APTT) reflected severe DIC. We suspected severe complications of the influenza virus infection based on the clinical symptoms. However, the secondary influenza test was negative, as well as the dengue test. Abdominal computed tomography (CT) scanning was arranged to rule out an intraabdominal infection. Even with early administration of ceftriaxone and levofloxacin, progressive cyanosis at bilateral fingers and lips was found along with dyspnea. The patient experienced a sudden cardiac arrest. Despite cardiopulmonary resuscitation (CPR), he expired. Subsequently, the blood culture report showed Neisseria meningitidis. This result was confirmed by the Centers for Disease Control and Prevention (CDC), who detected Neisseria meningitidis serogroup B.

Table 1. The laboratory evaluation of this patient.

\begin{tabular}{|c|c|c|c|c|c|c|c|}
\hline \multirow{2}{*}{ Variables } & \multirow{2}{*}{ Normal Range } & \multicolumn{2}{|c|}{ Patient Data } & \multirow{2}{*}{ Variables } & \multirow{2}{*}{ Normal Range } & \multicolumn{2}{|c|}{ Patient Data } \\
\hline & & Day 1 & Day 2 & & & Day 1 & Day 2 \\
\hline White cell count & $3.9-10.6(\times 103 / \mu \mathrm{L})$ & 8.69 & 2.19 & Blood urine nitrogen & $7-25$ mg/dL & 17 & 26 \\
\hline Hemoglobin & $13.5-17.5 \mathrm{~g} / \mathrm{dL}$ & 15.1 & 15.5 & Creatinine & $0.7-1.3 \mathrm{mg} / \mathrm{dL}$ & 1.1 & 2.7 \\
\hline Platelet count & $150-400(\times 103 / \mu \mathrm{L})$ & 152 & 35 & Sodium & $136-145 \mathrm{mmol} / \mathrm{L}$ & 133 & 134 \\
\hline Band & $0-3 \%$ & 1.0 & 6.0 & Potassium & $3.5-5.1 \mathrm{mmol} / \mathrm{L}$ & 3.6 & 4.0 \\
\hline Monocytes & $2-10 \%$ & 1.0 & 4.0 & Glucose & $70-100 \mathrm{mg} / \mathrm{dL}$ & 90 & 99 \\
\hline Neutrophils & $40-45 \%$ & 95.0 & 62.0 & ALT & 7-52 U/L & - & 28 \\
\hline Lymphocytes & $20-45 \%$ & 3.0 & 24.0 & AST & 13-39 U/L & - & 44 \\
\hline Eosinophils & $1-6 \%$ & 0.0 & 1.0 & Total bilirubin & $0.3-1.0 \mathrm{mg} / \mathrm{dL}$ & - & 2.08 \\
\hline N. RBC & $0-0 \%(/ 100 \mathrm{WBC})$ & 0.0 & 7.0 & C-reactive protein & $<1.0 \mathrm{mg} / \mathrm{dL}$ & 0.23 & 10.33 \\
\hline Metamyelocytes & $0-0 \%$ & 0.0 & 2.0 & Lactic acid & $0.5-2.2 \mathrm{mg} / \mathrm{dL}$ & - & 11.3 \\
\hline PT & $8-12$ s & - & 28.2 & Hs-troponin I & $<17.5 \mathrm{pg} / \mathrm{mL}$ & - & 64.8 \\
\hline APTT & $23.9-35.5 \mathrm{~s}$ & - & 166.2 & Influenza test & Negative & Negative & Negative \\
\hline INR & & - & 2.77 & Dengue test* & Negative & Negative & Negative \\
\hline Venous gas pH & 7.31-7.41 & - & 7.239 & & & & \\
\hline Venous gas $\mathrm{pCO}_{2}$ & $41-51 \mathrm{~mm} \mathrm{Hg}$ & - & 41.7 & & & & \\
\hline Venous gas $\mathrm{pO}_{2}$ & 80-100 mm Hg & - & 33.6 & & & & \\
\hline $\begin{array}{l}\text { Venous gas } \\
\mathrm{HCO}_{3}^{-}\end{array}$ & $22-26 \mathrm{mmol} / \mathrm{L}$ & - & 17.4 & & & & \\
\hline
\end{tabular}

N. RBC: Nucleated red blood cells; PT: Prothrombin time; APTT: Activated partial thromboplastin time; ALT: Alanine aminotransferase; AST: Aspartate aminotransferase; Dengue test*: included NS1 antibodies, IgM, and IgG; INR: international normalized ratio. 


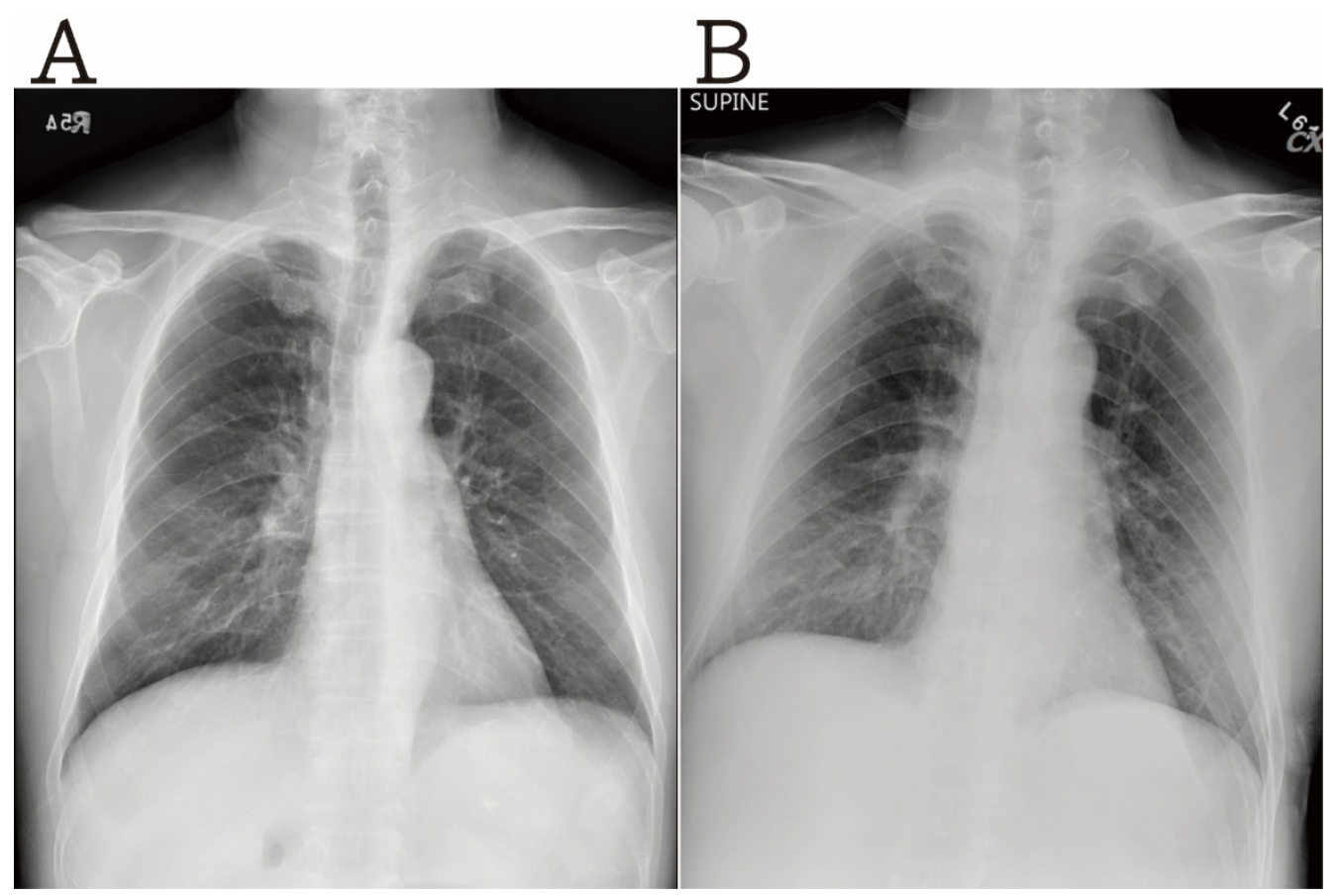

Figure 1. The chest $X$-ray revealed mild increased bilateral lung markings without a significant pneumonia patch (A) initially (B) after one day.

\section{Discussion}

Neisseria meningitidis is a gram-negative human-specific pathogen classified into thirteen serotypes [3]. Serotypes A, B, C, Y, and W135 are the major disease-causing serogroups. In the United States, serotype B causes about one-third of all invasive diseases [4]. The clinical manifestations of Neisseria meningitidis could range from transient fever with non-specific symptoms to invasive and fulminant diseases, such as meningitis and meningococcemia. The clinical presentation of meningitis includes the classic symptoms: headache, neck stiffness, and fever. Other non-specific symptoms are nausea, vomiting, myalgia, and poor appetite. In the present case, only flu-like symptoms were found. He denied any significant symptoms of urinary tract infection, acute intraabdominal infection, and meningitis. In a study by Rodrigo Siqueira Batista et al. [5], symptoms such as sore throat, coryza, cough, and otalgia were found after the incubation period. The classic symptoms occurred only in $44 \%$ of patients and were also more common in Streptococcus pneumoniae infection [6]. These atypical symptoms could be misdiagnosed as a viral infection, especially influenza. Skin rash, such as a petechial, maculopapular eruption, an ecchymotic lesion, and purpura fulminans are rare hallmarks of an invasive meningococcal disease. In the current patient, skin rash developed within $24 \mathrm{~h}$, and the condition was compatible with the previous data which reflected a worse prognosis. Other clinical features of worse prognosis included extreme age, primary meningococcal pneumonia, shock status, loss of consciousness, and seizures [7]. In addition, disease-promoting comorbidities such as immunosuppression could lead to missing the important symptoms or induce sepsis progression. In a report by Nicolas Paleiron et al. [8], a patient with a fulminant Neisseria meningitidis B infection was found with multiple myeloma, illustrating the importance of immunosuppression in Neisseria meningitidis infection. However, this patient did not have significant immunosuppressive diseases.

Purpura fulminans was characterized as vascular thrombosis with hemorrhagic necrosis of the skin with formation of bullae and vesicles. The necrosis can follow with extension into the subcutaneous tissue, muscles and bones. These skin rashes were clinical indicators of the potential bleeding complications secondary to thrombocytopenia and disseminated intravascular coagulation (DIC). Progressive meningococcal sepsis also induced WFS, which is defined as an acute adrenal failure related to acute hemorrhagic necrosis of the adrenal glands. The typical symptoms of WFS include 
fever, maculopapular skin rash, petechiae, ecchymosis, cyanosis of the extremities, and shock resulting from severe sepsis via coagulopathy [9]. It could also be caused by other bacteria, such as Proteus Mirabilis, Pseudomonas aeruginosa, Streptococcus pneumoniae, Mycobacterium tuberculosis, and Haemophilus influenzae $[10,11]$. WFS is a catastrophic syndrome, and it is difficult to be diagnosed in the clinical course due to hypotension and quick progression to shock. Adrenal hemorrhage is usually confirmed during the autopsy [12]. In the present case, an abdominal CT scan was arranged for high suspicion of WFS due to the typical clinical features. However, the progressive shock status could not be corrected even after aggressive resuscitation.

The pathophysiology of a meningococcemia-induced WFS remained unclear. In a study by Pathan et al. [13], Neisseria meningitidis may adhere to non-ciliated epithelial cells and avoid host immune systems by releasing the IgA protease, presenting with asymptomatic nasopharyngeal colonization. After invasion into the bloodstream, the endotoxin of Neisseria meningitidis may trigger immune cell activation, including the monocytes and neutrophils. Neutrophils can release the proteases causing endothelial injury. Monocytes may promote cytokines to induce the systemic inflammatory response syndrome. In addition, the endotoxin may also activate the complement system and dysregulate the coagulation factors. According to a summary by Chang et al. [14], these are effects of dysregulation of the immune system causing endotheliopathy, leading to the activation of the inflammatory pathway and the microthrombotic pathway. This causes a cytokine storm, disseminated intravascular microthrombosis, and endotheliopathy-associated vascular microthrombotic disease. Finally, the patients may present with multiple organ failure and thrombotic thrombocytopenic purpura-like syndrome, such as WFS and purpura fulminans (Figure 2).

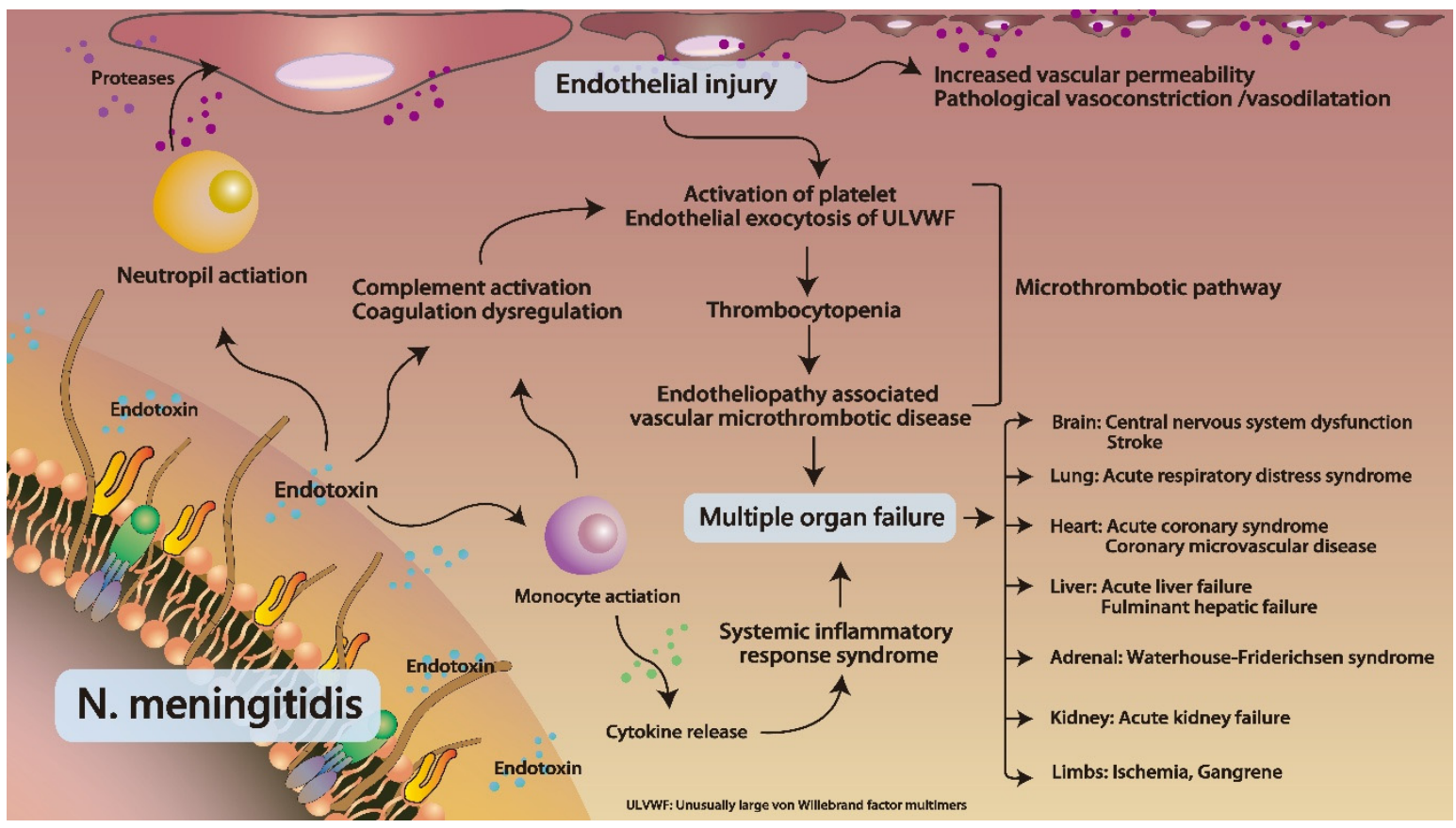

Figure 2. A schematic diagram illustrating the detailed pathophysiological mechanism between septicemia and disseminated intravascular coagulation in Neisseria meningitidis infection. Adapted from Chang [14,15] and Pathan et al. [13].

The prognosis of Neisseria meningitidis infection depends on its severity and treatment. The mortality of WFS is approximately $20 \%$, rising to more than $50 \%$ when there is shock. Early diagnosis and timely treatment of meningococcal sepsis is important to reduce the mortality. In pediatric or adult patients with an invasive meningococcal disease, the third generation of cephalosporin, ceftriaxone, was suggested to be the antibiotic of choice for Neisseria meningitidis infection. If patient was intolerant or allergic to beta-lactam antibiotics, chloramphenicol was an acceptable alternative medication [16]. 
In a severe invasive meningococcal disease, timely correction of the shock status is a critical step including rapid stabilization of the airways and breathing, intravenous access, and adequate fluid resuscitation [17]. Corticosteroids were considered for WFS due to the adrenal insufficiency status. Hydrocortisone $200 \mathrm{mg}$ daily has been recommended. In the current case, the flu-like symptoms without significant infection signs may have contributed to different diagnosis. When the skin rash erupted and progressive shock status occurred, Neisseria meningitidis-induced WFS was highly suspected. Despite our attempt to resuscitate, the fulminant sepsis could not be corrected even with administration of ceftriaxone. We present a rare case of Neisseria meningitidis-induced WFS to highlight an uncommon but important differential diagnosis that should be kept in mind in patients with a petechial, maculopapular eruption, or an ecchymotic lesion. Early management can prevent the poor clinical prognosis.

Author Contributions: Author Contributions: Conceptualization, G.-T.Y., J.-Y.K., C.-S.C. and M.-Y.W. Writing-Original Draft Preparation, M.-Y.W., P.-C.L. and C.-Y.T.; Writing-Review \& Editing, P.-Y.C., M.-Y.W. and J.-Y.K.; Visualization, M.-Y.W. All authors have read and agreed to the published version of the manuscript.

Funding: This study was supported by a grant from the Taipei Tzu Chi Hospital (TCRD-TPE-109-02, TCRD-TPE-109-03).

Conflicts of Interest: The authors declare no conflict of interest.

\section{References}

1. Ventura, F.; Bonsignore, A.; Portunato, F.; Orcioni, G.F.; Varnier, O.E.; De Stefano, F. A fatal case of streptococcal and meningococcal meningitis in a 2-years-old child occurring as Waterhouse-Friderichsen Syndrome. J. Forensic Leg. Med. 2013, 20, 678-682. [CrossRef] [PubMed]

2. Doherty, S. Fatal pneumococcal Waterhouse-Friderichsen syndrome. Emerg. Med. 2001, 13, $237-239$. [CrossRef] [PubMed]

3. Kingery, F.A. Waterhouse-Friderichsen Syndrome. JAMA 1965, 192, 569. [PubMed]

4. Tondella, M.L.C.; Popovic, T.; Rosenstein, N.E.; Lake, D.B.; Carlone, G.M.; Mayer, L.W.; Perkins, B.A. Distribution of Neisseria meningitidisSerogroup B Serosubtypes and Serotypes Circulating in the United States. J. Clin. Microbiol. 2000, 38, 3323-3328. [CrossRef] [PubMed]

5. Batista, R.S.; Gomes, A.P.; Gazineo, J.L.D.; Miguel, P.S.B.; Santana, L.A.; Oliveira, L.; Geller, M. Meningococcal disease, a clinical and epidemiological review. Asian Pac. J. Trop. Med. 2017, 10, 1019-1029. [CrossRef] [PubMed]

6. Van De Beek, D.; Spanjaard, L.; Vermeulen, M.; De Gans, J.; Weisfelt, M.; Reitsma, J.B. Clinical Features and Prognostic Factors in Adults with Bacterial Meningitis. N. Engl. J. Med. 2004, 351, 1849-1859. [CrossRef] [PubMed]

7. McGill, F.; Heyderman, R.S.; Panagiotou, S.; Tunkel, A.R.; Solomon, T. Acute bacterial meningitis in adults. Lancet 2016, 388, 3036-3047. [CrossRef]

8. Paleiron, N.; BaNguyen, V.; Commandeur, D.; Déserts, M.D.D.; Désidéri-Vaillant, C.; Sapin-Lory, J.; Valero, E.; Nicolas, X. Fulminant Neisseria meningitidis B to revealing multiple myeloma. Ann. Biol. Clin. (Paris) 2012, 70, 477-479. [CrossRef] [PubMed]

9. Zeldenrust, G.M.J.; van Suylen, R.J.; Ramakers, B.P.C. The Waterhouse-Friderichsen Syndrome. Ned. Tijdschr. Voor Geneeskd. 2018, 162, D2344.

10. Heitz, A.F.N.; Hofstee, H.M.A.; Gelinck, L.B.S.; Puylaert, J.B. A Rare Case of Waterhouse-Friderichsen Syndrome during Primary Varicella Zoster Infection. Neth. J. Med. 2017, 75, 351-353. [PubMed]

11. Spagnolo, E.V.; Mondello, C.; Roccuzzo, S.; Stassi, C.; Cardia, L.; Grieco, A.; Raffino, C. A unique fatal case of Waterhouse-Friderichsen syndrome caused by Proteus mirabilis in an immunocompetent subject. Medicine 2019, 98, e16664. [CrossRef] [PubMed]

12. Verzeletti, A.; Bonfanti, C.; Leide, A.; Azzalini, E.; De Francesco, M.A.; Piccinelli, G.; De Ferrari, F. Streptococcus Pneumoniae Detection Long Time After Death in a Fatal Case of Waterhouse-Friderichsen Syndrome. Am. J. Forensic Med. Pathol. 2016, 38, 1. [CrossRef] [PubMed]

13. Pathan, N.; Faust, S.N.; Levin, M. Pathophysiology of meningococcal meningitis and septicaemia. Arch. Dis. Child. 2003, 88, 601-607. [CrossRef] [PubMed] 
14. Chang, J.C. Sepsis and septic shock: Endothelial molecular pathogenesis associated with vascular microthrombotic disease. Thromb. J. 2019, 17, 10. [CrossRef] [PubMed]

15. Chang, J.C. TTP-like syndrome: Novel concept and molecular pathogenesis of endotheliopathy-associated vascular microthrombotic disease. Thromb. J. 2018, 16, 20. [CrossRef] [PubMed]

16. Steadman, E.; Raisch, D.W.; Bennett, C.L.; Esterly, J.S.; Becker, T.; Postelnick, M.; McKoy, J.M.; Trifilio, S.; Yarnold, P.R.; Scheetz, M.H. Evaluation of a Potential Clinical Interaction between Ceftriaxone and Calcium. Antimicrob. Agents Chemother. 2010, 54, 1534-1540. [CrossRef] [PubMed]

17. Van de Beek, D.; Brouwer, M.; Hasbun, R.; Koedel, U.; Cynthia, G. Whitney, and Eelco Wijdicks. "Community-Acquired Bacterial Meningitis." Nature reviews. Dis. Primers 2016, 2, 16074. [CrossRef] [PubMed]

(C) 2020 by the authors. Licensee MDPI, Basel, Switzerland. This article is an open access article distributed under the terms and conditions of the Creative Commons Attribution (CC BY) license (http://creativecommons.org/licenses/by/4.0/). 